\title{
XMM-Newton observation of the ULIRG NGC 6240
}

\section{The physical nature of the complex Fe $\mathrm{K}$ line emission}

\author{
Th. Boller ${ }^{1}$, R. Keil ${ }^{1}$, G. Hasinger ${ }^{1}$, E. Costantini ${ }^{1}$, R. Fujimoto ${ }^{2}$, N. Anabuki ${ }^{2}$, I. Lehmann ${ }^{1}$, and L. Gallo ${ }^{1}$ \\ 1 Max-Planck-Institut für extraterrestrische Physik, Postfach 1312, 85741 Garching, Germany \\ 2 Institute of Space and Astronautical Science, 3-1-1 Yoshinodai, Sagamihara, Kanagawa 229-8510, Japan
}

Received 22 April 2003 / Accepted 30 July 2003

\begin{abstract}
We report on an XMM-Newton observation of the ultraluminous infrared galaxy NGC 6240. The 0.3-10 keV spectrum can be successfully modelled with: (i) three collisionally ionized plasma components with temperatures of about 0.7, 1.4, and $5.5 \mathrm{keV}$; (ii) a highly absorbed direct power-law component; and (iii) a neutral $\mathrm{Fe} \mathrm{K} \alpha$ and $\mathrm{K} \beta$ line. We detect a significant neutral column density gradient which is correlated with the temperature of the three plasma components. Combining the $X M M$-Newton spectral model with the high spatial resolution Chandra image we find that the temperatures and the column densities increase towards the center.

With high significance, the Fe K line complex is resolved into three distinct narrow lines: (i) the neutral $\mathrm{Fe} \mathrm{K} \alpha$ line at $6.4 \mathrm{keV}$; (ii) an ionized line at about $6.7 \mathrm{keV}$; and (iii) a higher ionized line at $7.0 \mathrm{keV}$ (a blend of the Fe XXVI and the Fe K $\beta$ line). While the neutral Fe K line is most probably due to reflection from optically thick material, the Fe XXV and Fe XXVI emission arises from the highest temperature ionized plasma component.

We have compared the plasma parameters of the ultraluminous infrared galaxy NGC 6240 with those found in the local starburst galaxy NGC 253. We find a striking similarity in the plasma temperatures and column density gradients, suggesting a similar underlying physical process at work in both galaxies.
\end{abstract}

Key words. galaxies: active - galaxies: individual: NGC 6240 - galaxies: starburst - X-rays: galaxies

\section{Introduction}

The Infrared Astronomical Satellite (IRAS) mission (Neugebauer et al. 1984) resulted in the discovery of a large number of galaxies in the local universe $(z<0.3)$ with extraordinarily high infrared luminosities $\left(L_{\mathrm{IR}} \geq 10^{12} L_{\odot}\right.$ for $H_{0}=75 \mathrm{~km} \mathrm{~s}^{-1} \mathrm{Mpc}^{-1}$; see Sanders \& Mirabel 1996 for a review). The number density of these Ultraluminous InfraRed Galaxies (ULIRGs) exceeds that of optically selected Seyfert galaxies and QSOs with comparable bolometric luminosities (Soifer et al. 1987; Sanders et al. 1988a,b; Sanders \& Mirabel 1996) by a factor of $\sim 1.5-2$ (Sanders et al. 1999). Some of the most fundamental issues regarding ULIRGs are: the nature of the power source (strong starburst and/or a dust-enshrouded AGN); and the relative contribution to their bolometric luminosity. The capability of X-rays to penetrate high absorbing material makes X-ray observations essential in attempting to understand the physical processes at work in ULIRGs. A considerable portion of ULIRGs have been found to contain a hard X-ray source, highly absorbed by a molecular torus, which indicates the presence of a hidden AGN (Mitsuda 1995; Brandt et al. 1997; Kii et al. 1997; Vignati et al. 1999; Braito et al. 2003; Ptak et al. 2003; Franceschini et al. 2003).

Send offprint requests to: Th. Boller, e-mail: bol@mpe.mpg.de
NGC 6240 (IRAS 16504+0228) is perhaps one of the most interesting and best studied objects in this class. Although it is slightly less luminous $\left(L_{\mathrm{FIR}} \simeq 6 \times 10^{11} L_{\odot}\right.$ for $H_{0}=$ $75 \mathrm{~km} \mathrm{~s}^{-1} \mathrm{Mpc}^{-1}$; Genzel et al. 1998) than the typical IR luminosity used to define a ULIRG, it exhibits all of the ULIRG characteristics, and it is thus studied as a prototype of this class of sources. Optical/IR observations show a complex system with a disturbed morphology consisting of two gravitationally interacting nuclei (Fosbury \& Wall 1979; Fried \& Schulz 1983). An IS O observation of NGC 6240 (Genzel et al. 1998) favors a starburst-dominated power source, in which the high infrared emission arises from warm, absorbing dust, surrounding the inner parts of the galaxy. Early X-ray observations of NGC 6240 with ROSAT (Schulz et al. 1998) revealed an exceptionally high X-ray luminosity $\left(L_{0.1-2.0 \mathrm{keV}} \sim 10^{10} L_{\odot}\right)$, which further confirmed the importance of NGC 6240 in understanding the AGN-starburst connection. Therefore, considerable effort has been made to analyze the spectral and spatial properties using different X-ray satellites. Moreover, NGC 6240 can be considered as the prototype objects of highly absorbed AGN in the local universe (Hasinger 2003).

First ROSAT observations showed that a hot, diffuse gas model in combination with a power-law component can give a good description of the (0.1-2.4) keV energy band spectrum 
(Schulz et al. 1998). The power-law component was interpreted as evidence for a hidden AGN in NGC 6240. A luminous extended soft X-ray component was reported by Komossa et al. (1998) using ROSAT HRI observations. This emission should be responsible for at least $60 \%$ of the $0.1-2.4 \mathrm{keV}$ luminosity (within a region of $5^{\prime \prime}$ radius from the center). The combination of the spatial extent and the Raymond-Smith-like thermal spectrum led to the idea that the origin of the soft X-rays were strong thermal processes indicative of a powerful, X-ray luminous starburst.

According to data from ROSAT and ASCA, Iwasawa \& Comastri (1998) reported the detection of a highly absorbed hard X-ray source in NGC 6240. They pointed out that the soft X-ray spectrum $(\sim 0.5-2.0 \mathrm{keV})$ could be described by a superposition of two optically thin thermal plasmas with different temperatures $\left(k T_{1} \simeq 0.2-0.6 \mathrm{keV}\right.$ and $\left.k T_{2} \simeq 1 \mathrm{keV}\right)$ and an $N_{\mathrm{H}}$ value roughly an order of magnitude higher than the Galactic value of $N_{\mathrm{H}, \mathrm{gal}}=5.8 \times 10^{20}$ atoms $\mathrm{cm}^{-2}$. At higher energies $(\sim 3-10 \mathrm{keV}) A S C A$ observed an extremely hard continuum slope accompanied by the presence of a strong Fe K $\alpha$ complex (Mitsuda 1995; Iwasawa \& Comastri 1998). This can be interpreted in terms of a luminous AGN completely hidden by Compton-thick material, whose emitted light is reflected into the line-of-sight (e.g. George et al. 1990). In addition, the Fe complex could be well explained by a superposition of two Gaussian lines at $E_{1}=(6.44 \pm 0.04) \mathrm{keV}$ and $E_{2}=(6.87 \pm 0.05) \mathrm{keV}$, this latter was interpreted as a strong hint for a highly ionized reflecting material.

Vignati et al. (1999) and Ikebe et al. (2001) investigated NGC 6240 by using data from BeppoSAX and RXTE, respectively. Both authors assert the existence of an AGN component directly related to a Compton thick absorber (with $N_{\mathrm{H}} \simeq$ $2 \times 10^{24}$ atoms $\mathrm{cm}^{-2}$ ).

Recent Chandra observations have spatially resolved the emission from the two nuclei and the surrounding starbursts at $\mathrm{X}$-rays. The presence of the neutral $\mathrm{Fe} \mathrm{K} \alpha$ lines and the hard power-law-like X-ray emission from both nuclei was used to infer that both nuclei of the galaxy harbours an AGN (Komossa et al. 2003). In addition, they found residua in their spectral fits at energies above the neutral $\mathrm{Fe} \mathrm{K}$ line, which they interpreted as possible emission from $\mathrm{H}$-like $\mathrm{Fe}$ from the nuclear regions.

This paper contains the analysis of the X-ray data of NGC 6240 taken with XMM-Newton (Jansen 1999). The data analysis and details of the XMM-Newton observations are described in Sect. 2. Section 3 contains the spectral fitting results. A comparison with the Chandra observations is given in Sect. 4. The summary of the XMM-Newton results on NGC 6240 is given in Sect. 5. We use a Hubble constant of $H_{0}=50 \mathrm{~km} \mathrm{~s}^{-1} \mathrm{Mpc}^{-1}$ and $q_{0}=0$ throughout.

\section{Technical details: $X M M-N e w t o n$ observation and data processing}

NGC 6240 was observed with XMM-Newton for the first time during orbit 0144 on the 22nd of September, 2000, with all instruments functioning except for the RGS. The EPIC pn camera was operated in the full-frame mode and the EPIC MOS cameras were operated in the large-window mode. Each of the detectors used the medium filter. The exposure times were $25150 \mathrm{~s}$ and $24349 \mathrm{~s}$ for the pn and MOS, respectively. The second observation, with a shorter exposure time of about $18 \mathrm{ks}$, was performed during orbit 0413 (12th of March, 2002), with all instruments working in the same mode and with the same filters as in the first exposure. In addition, the RGS was functional and operated in the Spectro+Q mode, however the exposure time was too low to collect sufficient signal (raw exposure times: $18917 \mathrm{~s}$ for each of the RGS devices).

The MOS1 and MOS2 data sets from each observation were merged for this analysis. Single and double events have been selected and the resulting PHA file was grouped with a minimum of 20 counts per bin. After correcting the MOS data for background flaring events, the GTI corrected exposure times for MOS1 and MOS2 were $23381 \mathrm{~s}$ and $23601 \mathrm{~s}$ (first observation - orbit 0144) and $11318 \mathrm{~s}$ and $11601 \mathrm{~s} \mathrm{(sec-}$ ond observation - orbit 0413). This gives us a total of about $35 \mathrm{ks}$ worth of exposure time which is used in the analysis. Examining each observation separately and comparing them to the merged data set, we found no discrepancies in the spectral fitting parameters within the data uncertainties, justifying the merging process. In addition, the EPIC pn data sets were merged in a similar fashion (orbit 0144: 16255 s, orbit 0413: $9750 \mathrm{~s})$.

The EPIC MOS energy resolution (FWHM) is slightly better than that of the EPIC pn. Comparing Figs. 25 and 26 of the XMM-Newton User's Handbook (v2.1; Document number: XMM-PS-GM 14 ${ }^{1}$ ) one gets an energy resolution for the EPIC MOS cameras of about $140 \mathrm{eV}$ between 6 and $7 \mathrm{keV}$. For the EPIC pn camera the energy resolution ranges between 160 and $240 \mathrm{eV}$, but it is better for double events (upper curve of Fig. 26) and decreases for single events when considering on-axis sources. As we have used single and double events, the EPIC pn energy resolution ranges between 160 and $240 \mathrm{eV}$ (the exact value depends on the ratio between single and double events which is presently not available). In order to utilize as many photons as possible we have concentrated on the EPIC MOS data for subsequent spectral analyses (about twice as many photons than in the EPIC pn data). With the combined MOS $1 / 2$ photons from the two observations we collect in the $0.3-10 \mathrm{keV}$ energy range about $1.0 \times 10^{-3}$ photons $\mathrm{cm}^{-2} \mathrm{~s}^{-1} \mathrm{keV}^{-1}$ (compared to $5 \times 10^{-4}$ photons $\mathrm{cm}^{-2} \mathrm{~s}^{-1} \mathrm{keV}^{-1}$ for EPIC $\mathrm{pn}$ ). Nevertheless we use the EPIC pn data to independently check the EPIC MOS results for consistency.

The data were processed using the standard procedures of the XMM-Newton Science Analysis System (XMM-SAS ${ }^{2}$ ) version 20020413_2031-5.3.0 with default parameters for the EPIC chains. These event lists were calibrated with the latest available calibration files ${ }^{3}$.

Source and background counts were extracted from circular regions with radius of $70^{\prime \prime}$. The background photons were extracted from a source free region with the same radius. For the X-ray spectral analysis we have used XSPEC version 11.2

\footnotetext{
1 ftp://xmm.vilspa.esa.es/pub/A03/XMM_UHB.pdf

2 http://xmm.vilspa.esa.es/sas/

3 http://xmm.vilspa.esa.es/ccf/
} 


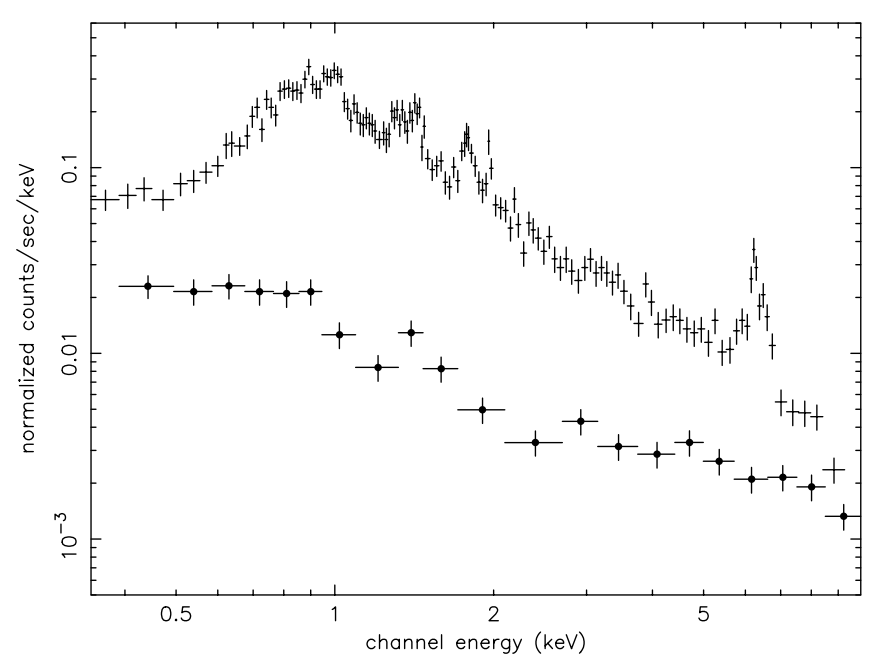

Fig. 1. The (0.3-10) keV spectrum of the combined source and background counts (upper curve) and the background counts (lower curve with the black dots) for the EPIC MOS1/2 data. The spectrum is not background dominated below $10 \mathrm{keV}$ and the Fe K line complex can be investigated with a high signal-to-noise ratio.

(Arnaud 1996) as well as FTOOLS version 5.2. The quoted errors on the derived best-fitting model parameters correspond to a $90 \%$ confidence level.

\section{Spectral analysis}

The combined MOS1/2 spectrum of NGC 6240 is not background dominated below $10 \mathrm{keV}$ (cf. Fig. 1); therefore, the energy range between $(0.3-10) \mathrm{keV}$ is analyzed with relatively good signal-to-noise.

\subsection{The soft energy spectrum}

\subsubsection{Line identification}

First, we searched for emission lines in the soft spectral region (0.3-3.5 keV). Figure 2 shows the EPIC MOS1/2 source spectrum and several identified emission lines. For illustration purposes we have included a $1-\mathrm{keV}$-model spectrum of a diffuse collisionally-ionized plasma, calculated using the APEC algorithm (Astrophysical Plasma Emission Code ${ }^{4}$; Smith et al. 2001) with variable abundances as provided by the vAPEC model in XSPEC (solid line). The abundances of $\mathrm{O}, \mathrm{Ne}, \mathrm{Mg}$, $\mathrm{Si}, \mathrm{S}$, and $\mathrm{Ar}$ were fixed to 10 times solar and the abundance of Fe was fixed to 0.01 times solar (the others were fixed to solar abundances). Pairs of emission lines, which are in different ionization states, can be resolved in the EPIC MOS1/2 spectrum. Moreover, higher energy emission line such as S XV and Ar XVII are also detected. The ratio of the emission line pairs and the emission lines at higher energies enable us to determine how many thermal plasma components are required to fit the soft energy spectrum.

\footnotetext{
${ }^{4}$ http://hea-www.harvard.edu/APEC/
}

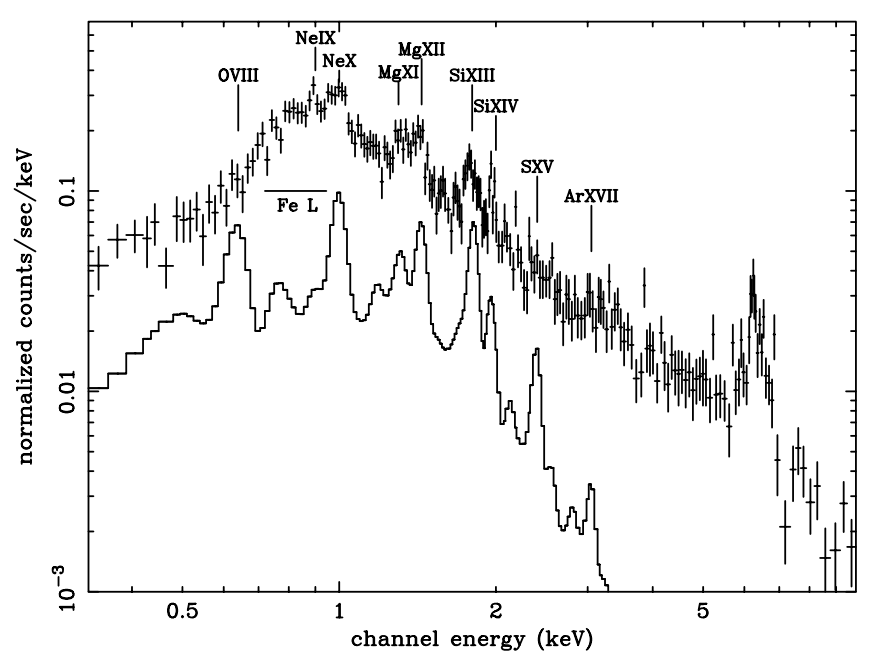

Fig. 2. EPIC MOS1/2 spectrum of NGC 6240 (upper curve). For illustration purposes a vAPEC model with a temperature of $1 \mathrm{keV}$ has been included (lower line).

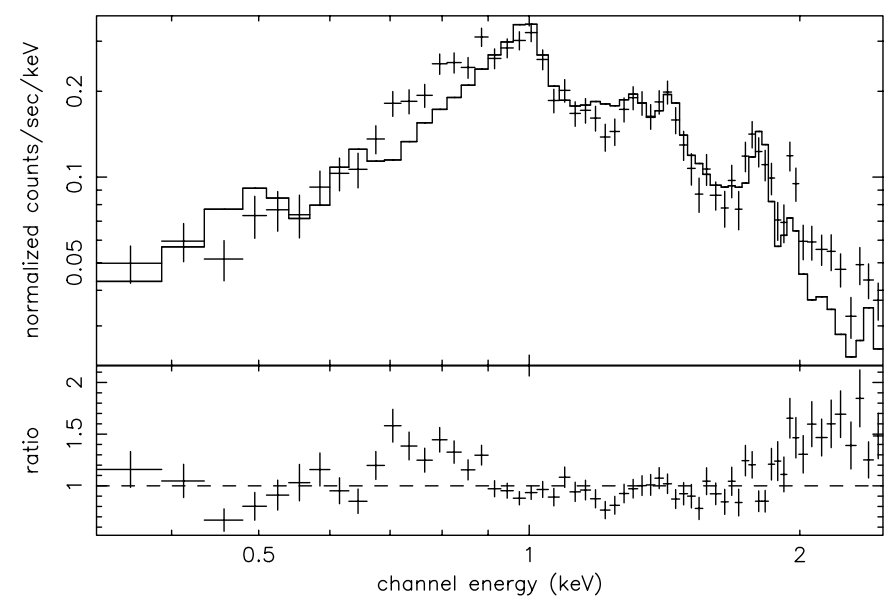

Fig. 3. Fitting only one vAPEC model to the EPIC MOS $1 / 2$ data in the (0.3-2.5) $\mathrm{keV}$ range, where $\mathrm{O}, \mathrm{Ne}, \mathrm{Mg}, \mathrm{Si}, \mathrm{S}$; the other $\alpha$ elements; and $\mathrm{Fe}-\mathrm{Ni}$ abundances have been left free. The resulting fit is statistically unacceptable.

\subsubsection{The presence of two distinct plasmas}

Applying only one vAPEC model to the EPIC MOS1/2 data with thawed abundance parameters for $\mathrm{O}, \mathrm{Ne}, \mathrm{Mg}, \mathrm{Si}, \mathrm{S}$, the $\alpha$ elements, and $\mathrm{Fe}-\mathrm{Ni}$, did not result in an acceptable fit. As can be seen in Fig. 3, significant residua remain in the Fe $\mathrm{L}$ emission line region around $0.8 \mathrm{keV}$, as well as in the $(1.0-1.5) \mathrm{keV}$ range $\left(\chi_{\mathrm{r}}^{2}=1.5\right)$. The observed Fe L bump, the flux ratio between two emission lines in different ionization states and the fit residuals all suggest the presence of a second thermal component.

We have added a second vAPEC component to the first model, which resulted in an acceptable fit $\left(\chi_{\mathrm{r}}^{2}=1.0\right)$. The significance of this new component was tested with the F-test. The second component is required with a significance greater than $5 \sigma$. The pn data explicitly confirm the EPIC MOS $1 / 2$ results. The best fit parameters of the two vAPEC components are reported in Table 1. 
Table 1. Spectral fit components to the (0.3-10) keV energy band. The individual Cols. 2 to 8 refer to the spectral components applied in the fit. The rows specify the measured values. The broad-band spectral energy distribution measured with these components is shown in Fig. 7. The foreground absorption has been fixed to the Galactic value. ${ }^{a}$ Note: for the first three components the normalization is given in units of $\left[10^{-14} /\left(4 \pi\left(D_{\mathrm{A}}(1+\mathrm{z})\right)^{2}\right) \int n_{\mathrm{e}} n_{\mathrm{H}} \mathrm{dV}\right]$, where $D_{\mathrm{A}}$ is the angular size distance to the source $(\mathrm{cm}), n_{\mathrm{e}}$ is the electron density $\left(\mathrm{cm}^{-3}\right)$, and $\mathrm{n}_{\mathrm{H}}$ is the hydrogen density $\left(\mathrm{cm}^{-3}\right)$. For the last five components the normalization is given in units of $\left[10^{-5}\right.$ photons $\left.\mathrm{cm}^{-2} \mathrm{~s}^{-1} \mathrm{keV}^{-1}\right]$ at $1 \mathrm{keV}^{\text {. The fluxes }}$ and luminosities are absorption corrected.

\begin{tabular}{lllllllll}
\hline \hline$(1)$ & $(2)$ & $(3)$ & $(4)$ & $(5)$ & $(6)$ & $(7)$ & $(8)$ & $(9)$ \\
\hline & vAPEC(1) & vAPEC(2) & APEC(3) & power-law & Fe K $\alpha$ & Fe XXV & Fe XXVI & Fe K $\beta$ \\
\hline$N_{\mathrm{H}}(\mathrm{fit})\left[10^{22} \mathrm{~cm}^{-2}\right]$ & $0.20 \pm 0.03$ & $0.4 \pm 0.03$ & $4.1 \pm 1.3$ & $100 \pm 20$ & - & - & - & - \\
$k T[\mathrm{keV}]$ & $0.66 \pm 0.03$ & $1.4 \pm 0.2$ & $5.5 \pm 1.5$ & - & - & - & - & - \\
$\mathrm{Norm}^{a}$ & $4.8 \pm 1.2$ & $2.5 \pm 1.5$ & $3.7 \pm 1.3$ & $6.0 \pm 3.0$ & $2.6 \pm 0.8$ & $1.8 \pm 0.7$ & $0.9 \pm 0.7$ & $0.3 \pm 0.1$ \\
$f\left[10^{-12} \mathrm{erg} \mathrm{cm}^{-2} \mathrm{~s}^{-1}\right]$ & $1.5 \pm 0.4$ & $0.7 \pm 0.4$ & $1.4 \pm 0.5$ & $40 \pm 20$ & $0.25 \pm 0.08$ & $0.19 \pm 0.07$ & $0.10 \pm 0.08$ & $0.03 \pm 0.01$ \\
$L\left[10^{42} \mathrm{erg} \mathrm{s}^{-1}\right]$ & $3.9 \pm 1.0$ & $1.9 \pm 1.1$ & $3.6 \pm 1.3$ & $100 \pm 50$ & $0.60 \pm 0.20$ & $0.50 \pm 0.20$ & $0.30 \pm 0.20$ & $0.08 \pm 0.02$ \\
Equivalent width $[\mathrm{eV}]$ & - & - & - & - & $300 \pm 100$ & $220 \pm 90$ & $120 \pm 90$ & $50 \pm 20$ \\
\hline
\end{tabular}

\subsection{The Fe K line complex}

The high throughput of XMM-Newton allows for precise measurements of the Fe $\mathrm{K}$ line complex compared to previous $\mathrm{X}$-ray missions. In this section we will demonstrate with high significance that the $\mathrm{Fe} \mathrm{K}$ line complex is resolved into three distinct lines. The line energies are given in the rest frame of the source.

\subsubsection{XMM-Newton detection of three distinct Fe K emission lines}

As a simple first approach the $5-7.2 \mathrm{keV}$ energy range has been fitted with a power-law model and two Gaussian lines (cf. Fig. 4, upper panel). The centroid line energies are found at $(6.5 \pm 0.3) \mathrm{keV}$ and $(6.68 \pm 0.02) \mathrm{keV}$. Strong residua remain at around $6.9 \mathrm{keV}$ and the fit is statistically unacceptable ( $\chi^{2}=30$ for 22 d.o.f.). We can exclude the possibility that the Fe line complex can be modelled by a narrow unresolved $6.4 \mathrm{keV}$ line and a blend of higher ionized lines (Fig. 4, middle panel; $\chi^{2}=30$ for 20 d.o.f.).

The strong residuals which remain at $6.8-6.9 \mathrm{keV}$ clearly are reduced by using a power-law plus three narrow unresolved Gaussian lines ( $\chi^{2}=15$ for 19 d.o.f., see Fig. 4 , bottom panel). The centroid line energies and corresponding 90 per cent coincidence ranges are $(6.41 \pm 0.02) \mathrm{keV},(6.68 \pm 0.02) \mathrm{keV}$ and $(7.01 \pm 0.04) \mathrm{keV}$. With high statistical significance, the XMMNewton data reveal for the first time the presence of three narrow Fe $\mathrm{K}$ lines.

\subsubsection{The possible physical origin of the ionized Fe $\mathrm{K}$ lines}

One plausible model to explain the presence of ionized $\mathrm{Fe} \mathrm{K}$ emission lines arises from the presence of a collisionally ionized plasma (using the APEC model in XSPEC). The plasma with a temperature of about $5.5 \mathrm{keV}$ produces the Fe XXV (at $6.68 \mathrm{keV}$ ) and Fe XXVI (6.93 keV) emission lines (cf. also the discussion of the broad band spectrum in Sect. 3.3). In the spectral fit for the Fe line complex we have also included the emission from the $\mathrm{Fe} \mathrm{K} \alpha$ line at $6.4 \mathrm{keV}$ and the $\mathrm{Fe} \mathrm{K} \beta$ line at $7.058 \mathrm{keV}$, each modelled with a Gaussian line. The normalization in the spectral fit of the line intensity of the $\mathrm{Fe} \mathrm{K} \beta$ line was set to $1 / 8.8$ of the $\mathrm{Fe} \mathrm{K} \alpha$ line intensity, as expected from atomic physics. Figure 5 shows the EPIC MOS $1 / 2$ spectral fitting results for the Fe line complex in NGC $6240\left(\chi_{\mathrm{r}}^{2}=1.1\right)$. The model components are displayed in Fig. 6.

In addition, we tested other possibilities to describe the origin of the ionized line emission in NGC 6240. First, we modelled the Fe $\mathrm{K}$ line complex by $\mathrm{He}$ - and $\mathrm{H}$-like emission from the accretion disk. Using the Ballantyne et al. (2001) model available within XSPEC we are unable to find a statistically acceptable fit. This is expected as BeppoSAX observations show that the direct AGN component is heavily absorbed and only dominates the spectrum above $8 \mathrm{keV}$ (cf. Fig. 3 of Vignati et al. 1999). Therefore, the putative He- and H-like emission from the accretion disk should remain undetectable.

Secondly, we substituted the APEC model by an absorbed power-law component plus three Gaussian lines. The fit to the $(0.3-10) \mathrm{keV}$ spectrum was statistically unacceptable $\left(\chi_{\mathrm{r}}^{2}=1.8\right)$ and is unable to explain the broad-band spectral energy distribution, nor the presence of ionized $\mathrm{Fe} \mathrm{K}$ lines.

Thirdly, fitting the broad-band spectrum with only two APEC components was statistically unacceptable. A third APEC component is required by the data. The two lower temperature APEC components have already been discussed in Sect. 3.1.2.

Colbert et al. (2002) have modelled the Fe $\mathrm{K}$ features in NGC 1068 from both optically thick and optically thin ionized reflection emission. The optically thick case was modelled using the PEXRIV model in XSPEC, the optically thin case by a power-law plus edge model. The authors find that the Compton reflection component provided by the optically thick model does give a better fit than the optically-thin reflection model. We have modelled the XMM-Newton spectrum by substituting the APEC model with the PEXRIV model (and by adding two Gaussian lines to model the ionized Fe $\mathrm{K}$ lines). This model does not provide an acceptable fit $\left(\chi_{\mathrm{r}}^{2}=4.6\right)$. The same holds for the power-law plus edge model for an optically thin warm 

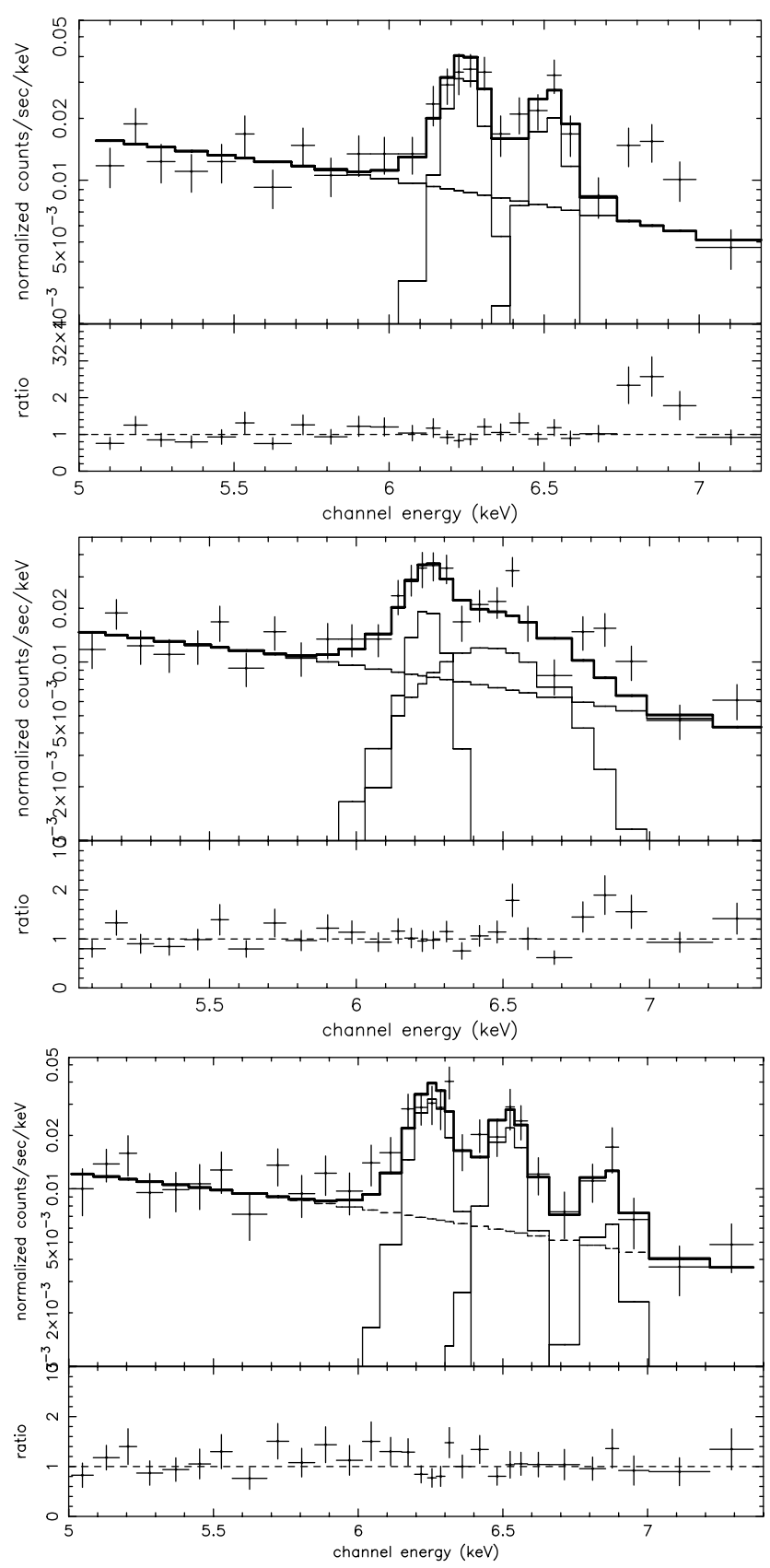

Fig. 4. Upper panel: spectral fit to the XMM-Newton combined MOS1/2 data of NGC 6240 in the Fe K line range. Fitting the Fe line complex with only two narrow lines and a power-law continuum results in a statistically unacceptable fit. Middle panel: Fitting the Fe line complex with a narrow neutral line and one broad line also result in a statistically unacceptable fit. Bottom panel: An acceptable fit is obtained when fitting three Gaussian lines above the power-law continuum. The centroid line energies are at $(6.41 \pm 0.02) \mathrm{keV},(6.68 \pm$ $0.02) \mathrm{keV}$ and $(7.01 \pm 0.04) \mathrm{keV}$. With high statistical significance, the XMM-Newton data reveal for the first time the presence of three narrow Fe K lines.

reflector. The XMM-Newton data do not require, within the available statistics, an additional ionized edge.

The collisionally ionized plasma provides the best spectral fitting results and appears the most likely explanation for these

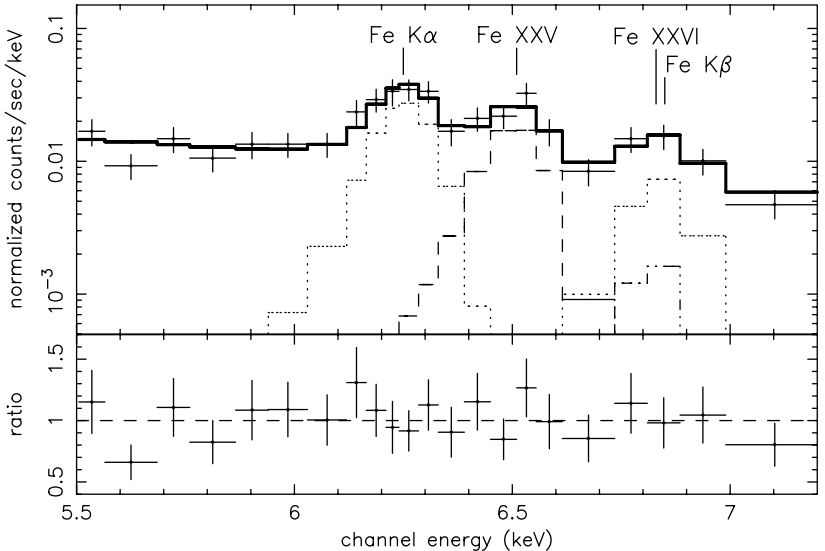

Fig. 5. Spectral fit to the XMM-Newton MOS1/2 data of NGC 6240 in the Fe $\mathrm{K}$ line range (with the identifications of the Fe lines). Emission from a collisionally ionized plasma has been assumed to model the ionized $\mathrm{Fe} \mathrm{K}$ line emission. The neutral $6.4 \mathrm{Fe} \mathrm{K} \alpha$ line has been modelled by a simple Gaussian line. We have also included the emission from the $\mathrm{Fe} \mathrm{K} \beta$ line where the ratio has been fixed to $1 / 8.8$ according to atomic physics. The Fe XXV and Fe XXVI lines originate from the hottest plasma component.

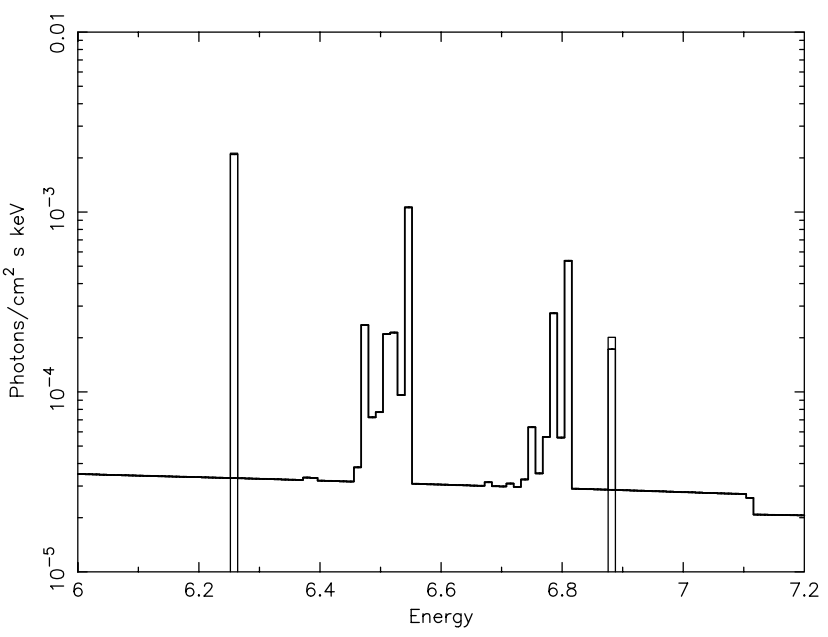

Fig. 6. Spectral model for the Fe K line complex. Two unresolved Fe lines $(\mathrm{K} \alpha$ and $\mathrm{K} \beta$ ) and emission from a collisionally ionized plasma (APEC model in XSPEC) can explain the emission from the $\mathrm{Fe} \mathrm{K}$ line complex in NGC 6240 (for line identifications see Fig. 5).

XMM-Newton observations (see Table 1 for the best fitting parameters).

\subsection{The broad-band X-ray spectrum}

The best fit to the $0.3-10 \mathrm{keV}$ XMM-Newton spectrum of NGC 6240 is obtained using: (i) three different plasma temperatures; (ii) a highly absorbed power-law component; and (iii) a neutral $\mathrm{Fe} \mathrm{K} \alpha$ and $\mathrm{K} \beta$ line (cf. Table 1 and Fig. 7). The broad-band fit with these spectral parameters results in an acceptable fit $\left(\chi_{\mathrm{r}}^{2}=0.93\right.$ for 190 d.o.f.). In the soft energy band the spectrum is dominated by the cold $(k T=0.7 \mathrm{keV})$ and medium $(k T=1.4 \mathrm{keV})$ plasma components. The medium component is intrinsically absorbed with an absorbing column of $(3.6 \pm 0.3) \times 10^{21} \mathrm{~cm}^{-2}$. For the $\alpha$ elements we find 


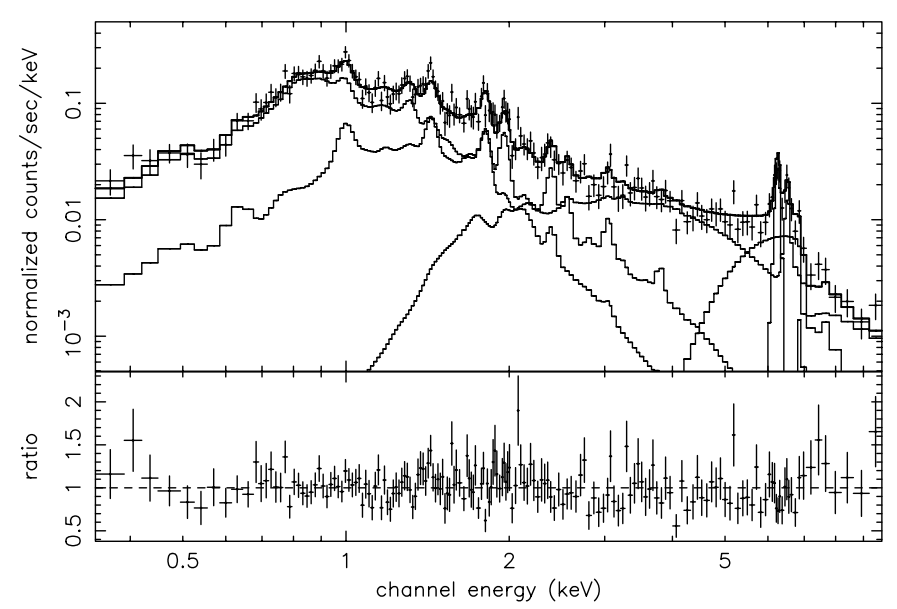

Fig. 7. Spectral fit to the merged MOS1/2 data of NGC 6240 in the $0.3-10 \mathrm{keV}$ energy range. In the soft energy band, a statistically acceptable fit is obtained by using a hot and cool plasma component, where the $\alpha$ and Fe-Ni abundances have been left free in the fit. The emission from a third hot plasma component is also required to reproduce the line ratio of the ionized line emission of the Fe K complex. A narrow unresolved $\mathrm{Fe} \mathrm{K} \alpha$ and $\mathrm{Fe} \mathrm{K} \beta$ line, with a relative ratio of $8.8: 1$, as expected from atomic physics, has been added to model. A highly absorbed $\left(N_{\mathrm{H}}=(1.0 \pm 0.3) \times 10^{24} \mathrm{~cm}^{-2}\right)$ power-law component with an photon index of 1.8 significantly improves the fit above about $7 \mathrm{keV}$. The fit to the $0.3-10 \mathrm{keV}$ band is statistically acceptable with the above mentioned components $\left(\chi^{2}=0.93\right.$ for 190 d.o.f.).

element abundances of $(0.15 \pm 0.08)$ relative to solar values. The Fe and nickel abundance is lower compared to that of the $\alpha$ elements with $(0.05 \pm 0.01)$; however, not significantly different. The cooler plasma component has an intrinsic column density of $(2.0 \pm 0.3) \times 10^{21} \mathrm{~cm}^{-2}$, and a temperature of $k T=(0.66 \pm 0.03) \mathrm{keV}$. The available photon statistics does not allow us to constrain the element abundances for the cooler plasma component; therefore, we have fixed the $\alpha$ and Fe and nickel abundances to that of the hotter plasma component, which results in a statistically acceptable fit for the soft energy region. However, this should be handled with caution. Finally, including a third hot plasma component and an absorbed power-law component allows us to explain the origin of the ionized, Fe XXV and Fe XXVI line emission which were discussed in detail in Sect. 3.2.2. This hottest plasma component has a temperature of $k T=(5.5 \pm 1.5) \mathrm{keV}$ and an intrinsic absorption of $(4.1 \pm 1.3) \times 10^{22} \mathrm{~cm}^{-2}$. The APEC components in this model are regarded as a description for starburst activity.

A heavily absorbed power-law is required by the $X M M$ Newton data. If neglected, significant residua remain above about $7 \mathrm{keV}$ and the fit is not statistically acceptable. We can confirm the BeppoSAX power-law with the XMM-Newton data at a $4 \sigma$ significance level (according to an F-test). The absorbing column density of $(1.0 \pm 0.3) \times 10^{24} \mathrm{~cm}^{-2}$ is a factor of two smaller than that found by BeppoSAX. We believe that the photon index can be better measured by BeppoSAX given the larger energy range over which it was determined. Therefore, we have fixed the power-law photon index to 1.8. The absorbed powerlaw is indicative of the direct emission from the accretion disk corona.

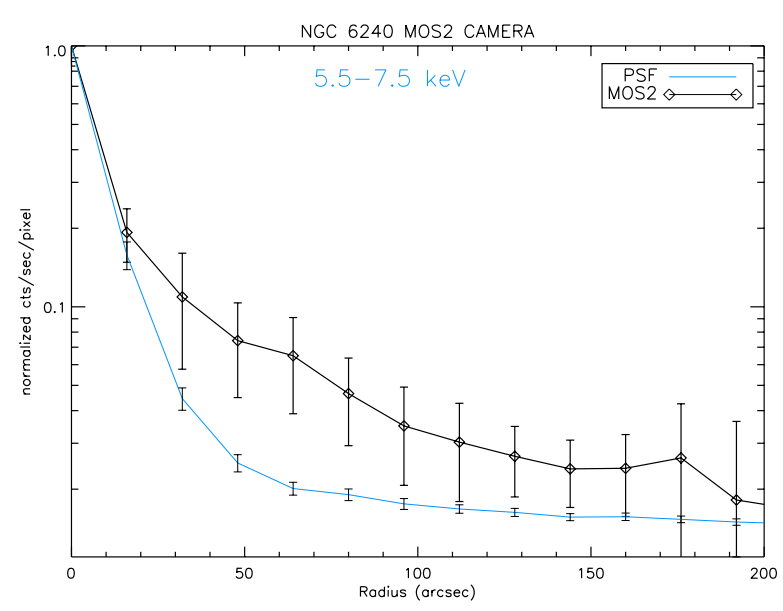

Fig. 8. The radial intensity profile of the EPIC MOS1/2 data compared to the instrumental point spread function (PSF; normalized to the maximum flux). The solid line shows the MOS PSF of $6.5 \mathrm{keV}$ and the photons have been selected in the $5.5-7.5 \mathrm{keV}$ range to match the emission from the Fe line complex. Extended emission is detected at distances of about $30^{\prime \prime}$ from the X-ray centroid position.

We also note that with the present statistics the fit is unable to independently determine the temperature of the hot plasma component, and the power-law photon index, and the column density.

In order to cross-check the EPIC MOS results obtained from the (v)APEC modelling with a different plasma code we substituted the three components with the corresponding MEKAL model: in the soft regime the two vMEKAL models and in the hard part of the spectrum a MEKAL model. We find no significant difference in the absorbing column densities and plasma temperatures with respect to the values listed in Table 1. For the $N_{\mathrm{H}}$ values we get $(0.15 \pm 0.02 ; 0.5 \pm 0.3$; and $3.5 \pm 1.1) \times 10^{22} \mathrm{~cm}^{-2}$. The corresponding plasma temperatures are $k T=(0.62 \pm 0.03) \mathrm{keV},(1.2 \pm 0.4) \mathrm{keV}$ and $(6.0 \pm 2.6) \mathrm{keV}$.

A second test was performed to compare the results of the combined EPIC MOS data with the data of EPIC pn. The resulting values $N_{\mathrm{H}}=(0.19 \pm 0.03) \times 10^{22}$ atoms $\mathrm{cm}^{-2}$ with $k T=(0.58 \pm 0.03) \mathrm{keV}$, and $N_{\mathrm{H}}=(0.4 \pm 0.1) \times 10^{22}$ atoms cm $^{-2}$ with $k T=(1.2 \pm 0.3) \mathrm{keV}$ for the two vMEKAL components are in good agreement to the values of the EPIC MOS data. The fitting with a MEKAL model yields in the values $N_{\mathrm{H}}=(3.8 \pm 2.0) \times 10^{22}$ atoms $\mathrm{cm}^{-2}$ and $k T=(5.0 \pm 1.9) \mathrm{keV}$.

\subsection{Comparison with local starbursts}

A striking similarity in the plasma parameters and absorbing columns is found between NGC 6240 and the prototype local starburst galaxy NGC 253. Pietsch et al. (2001) have analyzed the nuclear emission of NGC 253 with XMM-Newton. They found that the nuclear spectrum can be modelled by a three temperature plasma with the higher temperatures increasingly absorbed. The derived temperatures for the nuclear region are $0.56,0.92$ and $6.3 \mathrm{keV}$. The corresponding absorbing column densities are $(0.34,1.78,13.2) \times 10^{22}$ atoms $\mathrm{cm}^{-2}$. These temperatures and the corresponding absorbing column densities are remarkably similar to those found in NGC 6240. 

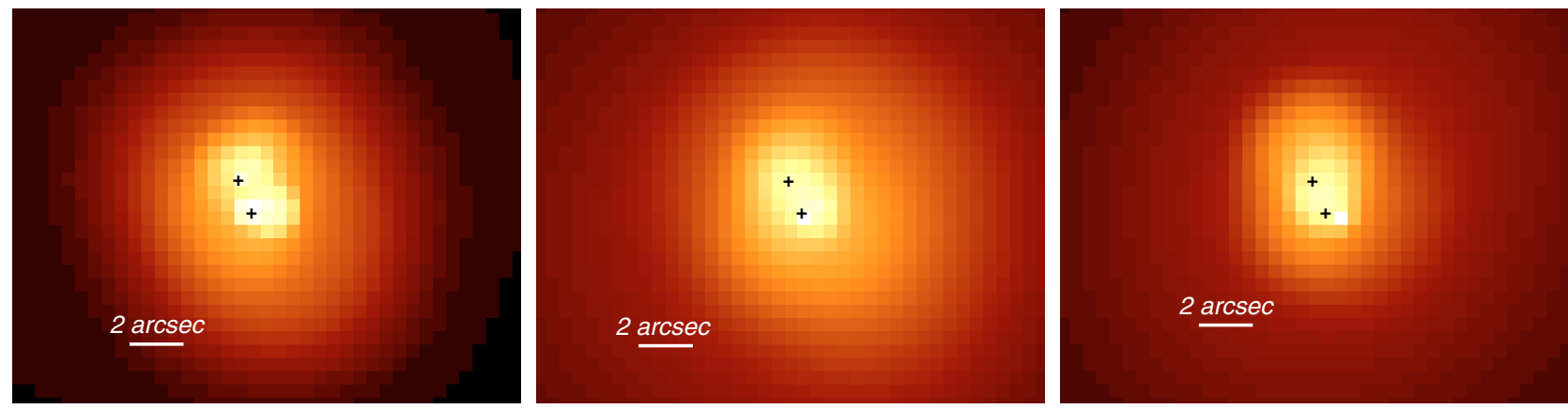

Fig. 9. We have binned the Chandra data in three different energy bands as seen by the XMM-Newton spectrum. The crosses mark the position of the two nuclei as detected with Chandra. The left panel shows emission from the neutral Fe K line (selected between 6.1 and $6.35 \mathrm{keV}$ in the observers frame, emission from the ionized lines (middle panel, energy range between 6.4 and $7 \mathrm{keV}$ ) as well as emission between 7 and $10 \mathrm{keV}$ from the highly absorbed power-law component (right panel). The spectral capabilities of XMM-Newton in combination with the high spatial resolution of the Chandra telescope gives us a more precise understanding of the nuclear region in NGC 6240.

In addition, Pietsch et al. detected He-like Fe $\mathrm{K}$ emission at $6.68 \mathrm{keV}$, similar to the detection in NGC 6240. The nondetection of the weaker H-like line in NGC 253 might be due to a lower signal-to-noise compared to NGC 6240. The authors attribute the presence of the high temperature component to emission from type Ib and type IIa SNRs. In addition, similar soft X-ray emission lines from the two cooler plasma components are detected in NGC 253, e.g. emission from Mg XI, Mg XII, Si XIII, Si XIV (cf. Sect. 4.1 of Pietsch et al.). The presence of different plasma components in the prototype local starburst galaxy NGC 253 support our spectral modelling, and further suggests that there may be a common underlying physical mechanism, which appear to be dominated by emission from type Ib and type IIa SNRs.

\section{Comparison with Chandra}

In the following we combine the high throughput of XMMNewton with the high spatial resolution of Chandra. While Chandra has resolved the nuclear region into two nuclei and diffuse emission components (Komossa et al. 2003), XMMNewton allows for X-ray spectroscopy with high statistics.

The inner 30" remain unresolved with XMM-Newton as obtained from the PSF. The PSF was created simulating a point source at the same position on the detector as NGC 6240, and at an energy centered at $6.5 \mathrm{keV}$, using the XMM science simulator software SciSim ${ }^{5}$. The energy selected corresponds to the mean energy of the Fe K line complex. Serendipitous sources (localized through the XMMSAS task emldetect) and out of time events were removed from the data. Each photon in the profile was corrected for vignetting and divided by the exposure map, to remove chip gaps and hot pixels. Since neither the simulated source nor NGC 6240 show any pile-up, the sources were normalized to the maximum flux. Using such a PSF will allow us to further investigate the spectral components around the two nuclei detected with Chandra. The data were accumulated in the (5.5-7.5) keV energy range for the combined MOS1/2 data. The radial intensity profile of the emission, together with the PSF are shown in Fig. 8. Due to the large width

\footnotetext{
${ }^{5}$ http://xmm.vilspa.esa.es/scisim/
}

of the PSF, the (PSF-uncorrected) extended emission appears to start at a distance greater than $30^{\prime \prime}$ from the center. The spectral analysis of the extended emission was extended up to a distance of $70^{\prime \prime}$. Beyond this distance the number of counts is too small to make a significant contribution to the statistics.

We have performed a spectral analysis of the inner $30^{\prime \prime}$ independent from the results obtained from the total emission $\left(0-70^{\prime \prime}\right)$. Interestingly, the spectral fit to the inner $30^{\prime \prime}$ is consistent with the spectral fit components and parameters obtained for the total emission (cf. Fig. 7) except for the flux emitted by the cold APEC component. Ninety-eight per cent of the $(0.3-10 \mathrm{keV})$ flux is emitted in the inner $30^{\prime \prime}$ core. The derived temperatures for the plasma components confirm the existence of different plasma contributions in the core region.

In the following we compare the XMM-Newton plasma components with the energy bands used in the high resolution Chandra image (Fig. 3 of Komossa et al. 2003). In the softest energy band (red coloured; $0.5-1.5 \mathrm{keV}$ ) the emission from the $0.7 \mathrm{keV}$ plasma component dominates the spectral energy distribution, with a contamination level of about 10 per cent from the $1.4 \mathrm{keV}$ component. In the (1.5-5) keV band (yellow and green coloured), we have a mixture of all three plasma components and cannot uniquely attribute a single temperature to the spatial location. Above $5 \mathrm{keV}$ the hot $5.5 \mathrm{keV}$ plasma component dominates the spectrum and is responsible for the emission from Fe XXV and Fe XXVI. This is confirmed by the Chandra observation; Komossa et al. (2003) note that the neutral Fe $\mathrm{K}$ line dominates the spectral energy distribution between (6-7) $\mathrm{keV}$, and noticed that some residua remain in their spectra which can be attributed to ionized Fe $\mathrm{K}$ emission. In addition, the XMM-Newton data reveal a strong column density gradient for the different plasma components for the inner region, decreasing outwards from about $4.1 \times 10^{22} \mathrm{~cm}^{-2}$ $(k T=5.5 \mathrm{keV})$, to $0.4 \times 10^{22} \mathrm{~cm}^{-2}(k T=1.4 \mathrm{keV})$, and down to $0.2 \times 10^{22} \mathrm{~cm}^{-2}(k T=0.7 \mathrm{keV})$.

\section{Summary}

XMM-Newton observations of the ULIRG NGC 6240 reveal: (1) For the first time, the presence of three distinct Fe $\mathrm{K}$ lines 
located at $(6.41 \pm 0.02) \mathrm{keV},(6.68 \pm 0.02) \mathrm{keV}$ and $(7.01 \pm$ $0.04) \mathrm{keV}$ (in the rest frame of the source).

(2) That the broad-band spectrum is successfully described by the emission from three different plasma components with temperatures of $(0.66 \pm 0.03) \mathrm{keV},(1.4 \pm 0.2) \mathrm{keV}$, and $(5.5 \pm 1.5) \mathrm{keV}$. The ionized lines can be explained by emission from the highest temperature plasma components resulting in emission from Fe XXV and Fe XXVI. The three plasma components are found at different locations in the Chandra image, showing that the temperature and the column density are increasing towards the center of NGC 6240.

(3) A strong column density gradient for the different plasma components increasing with temperature from $(0.20 \pm$ $0.03) \times 10^{22} \mathrm{~cm}^{-2}$, to $(0.40 \pm 0.03) \times 10^{22} \mathrm{~cm}^{-2}$, to $(4.1 \pm 1.3) \times$ $10^{22} \mathrm{~cm}^{-2}$.

(4) The presence of several emission lines in the soft energy band which is in agreement with previous ASCA measurements.

(5) A highly absorbed direct power-law component, confirming the BeppoSAX results.

(6) A striking similarity to the local starburst galaxy NGC 253. In both, NGC 253 and NGC 6240, three plasma components are required, and their temperatures and column density gradients are remarkably similar; hence suggesting a similar underlying physical process at work in both galaxies.

The spectral capabilities of XMM-Newton in combination with the high spatial resolution of the Chandra telescope gives us a more precise understanding of the nuclear region in NGC 6240 (cf. Fig. 9).

Acknowledgements. Based on observations obtained with XMMNewton, an ESA science mission with instruments and contributions directly funded by ESA Member States and the USA (NASA). We thank the anonymous referee for excellent suggestions and comments.

\section{References}

Arnaud K. A. 1996, Astronomical Data Analysis Software and Systems V, ed. G. Jacoby, \& J. Barnes, ASP Conf. Ser., 101, 17

Ballantyne, D. R., Iwasawa, K., \& Fabian, A. C. 2001, MNRAS, 323 506
Braito, V., Franceschini, A., Della Ceca, R., et al. 2003, A\&A, 398, 107

Brandt, W. N., Fabian, A. C., Takahashi, K., et al. 1997, MNRAS, 290, 617

Colbert, E. J. M., Weaver, K. A., Krolik, J. H., Mulchaey, J. S., \& Mushotzky, R. F. 2002, ApJ, 581, 182

Downes, D., Solomon, P. M., \& Radford, S. J. E. 1993, ApJ, 414, L13

Elvis, M., Wilkes, B. J., McDowell, J. C., et al. 1994, ApJS, 95, 1

Fosbury, R. A. E., \& Wall, J. V. 1979, MNRAS, 189, 79

Franceschini, A., Braito, V., Persic, M., et al. 2003, MNRAS, 343, 1181

Fried, J. W., \& Schulz, H. 1983, A\&A, 118, 166

Genzel, R., Lutz, D., Sturm, E., et al. 1998, ApJ, 498, 579

George, I. M., Nandra, K., \& Fabian, A. C. 1990, MNRAS, 242, 28

Hasinger, G. 2003, MPE Report, 281, 7

Ikebe, Y., Leighly, K., Tanaka, Y., et al. 2001, MNRAS, 316, 433

Iwasawa, K., \& Comastri, A. 1998, MNRAS, 297, 1219

Jansen, F. A. 1999, ESA Bulletin, 100, 9

Kii, T., Nakagawa, T., Fujimoto, R., et al. 1997, in X-Ray Imaging and Spectroscopy of Cosmic Hot Plasmas, ed. F. Makino, \& K. Mitsuda (Tokyo: Universal Acad. Press), 161

Komossa, S., Schulz, H., \& Greiner, J. 1998, A\&A, 334, 110

Komossa, S., Burwitz, V., Hasinger, G., et al. 2003, ApJ, 582, L15

Kormendy, J., \& Sanders, D. B. 1992, ApJ, 390, L53

Laurent-Muehleisen, S. A., Kollgaard, R. I., Ryan, P. J., et al. 1997, A\&AS, 122, 235

Magdziarz, P., \& Zdziarski, A. A. 1995, MNRAS, 273, 837

Mitsuda, K. 1995, Proc. 17th Texas Symp., ed. H. Böhringer, G. E. Morfill, \& J. E. Trümper (New York, NY: The NY Acad. of Sci.), 759,213

Neugebauer, G., Habing, H. J., van Duinen, R., et al. 1984, ApJ, 278, L1

Ptak, W., Roberts, T. P., Sako, D., et al. 2003, ApJ, 592, 782

Pietsch, V. A., Heckman, T., Levenson, N. A., et al. 2001, A\&A, 365, L174

Rieke, G. H., Cutri, R. M., Black, J. H., et al. 1985, ApJ, 290, 116

Sanders, D. B., Soifer, B. T., Elias, J. H., et al. 1988a, ApJ, 325, 74

Sanders, D. B., Soifer, B. T., Elias, J. H., Neugebauer, G., \& Matthews, K. 1988b, ApJ, 328, L35

Sanders, D. B., \& Mirabel, I. F. 1996, ARA\&A, 34, 749

Sanders, D. B., Surace, J. A., \& Ishida C. M. 1999, IAUS, 186, 289

Schulz, H., Komossa, S., Berghöfer, T. W., \& Boer, B. 1998, A\&A, 330,823

Smith, R. K., Brickhouse, N. S., Liedahl, D. A., \& Raymond, J. C. 2001, ApJ, 556, L91

Soifer, B. T., Sanders, D. B., Madore, B. F., et al. 1987, ApJ, 320, 238

Vignati, P., Molendi, S., Matt, G., et al. 1999, A\&A, 349, L57 